\title{
A MAC protocol for full exploitation of Directional Antennas in Ad-hoc Wireless Networks
}

\author{
Thanasis Korakis \\ Gentian Jakllari \\ Computer Engineering and Telecommunications Department \\ University of Thessaly \\ Glavani 37 \& 28 Octovriou, 38221, Volos, Greece \\ $+30-24210-74553$ \\ \{korakis, jakllari, leandros\}@inf.uth.gr
}

Leandros Tassiulas

\begin{abstract}
Directional antennas in ad hoc networks offer many benefits compared with classical omnidirectional antennas. The most important include significant increase of spatial reuse, coverage range and subsequently network capacity as a whole. On the other hand, the use of directional antennas requires new approach in the design of a MAC protocol to fully exploit these benefits. Unfortunately, directional transmissions increase the hidden terminal problem, the problem of deafness and the problem of determination of neighbors' location. In this paper we propose a new MAC protocol that deals effectively with these problems while it exploits in an efficient way the advantages of the directional antennas. We evaluate our work through simulation study. Numerical results show that our protocol offers significant improvement compared to the performance of omni transmissions.
\end{abstract}

\section{Categories and Subject Descriptors}

C.2.1 [Computer-Communication Networks]: Network Architecture and Design - Wireless Communication, Directional Antenna Systems; C.2.5 [Computer-Communication Networks]: Local and Wide-Area Network - Access Schemes.

\section{General Terms}

Performance, Design, Experimentation, Verification.

\section{Keywords}

Mobile Ad Hoc Networks, Directional Antenna Systems, Medium Access Control, IEEE 802.11.

\section{INTRODUCTION}

Ad-hoc wireless networks attract increasing interest in many application domains. There is a great interest for such networks in

Permission to make digital or hard copies of all or part of this work for personal or classroom use is granted without fee provided that copies are not made or distributed for profit or commercial advantage and that copies bear this notice and the full citation on the first page. To copy otherwise, or republish, to post on servers or to redistribute to lists, requires prior specific permission and/or a fee.

MobiHoc '03, June 1-3, 2003, Annapolis, Maryland, USA.

Copyright 2003 ACM 1-58113-684-6/03/0006...\$5.00. commercial applications, military applications as well as in environments where the presence of an infrastructure network is impossible or not affordable. The existing technology has equipped ad-hoc networks with omnidirectional antennas, forcing researchers to design Medium Access Control (MAC) protocols that control the wireless medium occupation taking under consideration the omnidirectional mode of transmission. The result of this effort is the design of many MAC protocols as MAC of IEEE 802.11 [5],[11] that seem to solve efficiently the problems that appear in this environment.

The electromagnetic energy of the signal in omnimode transmission is spread over a large region of space, while only a small portion of it is received by the intended receiver. Directional antennas is a technology that solves this problem. Using $M$ elements, this kind of antennas transmit in directional mode, which means that electromagnetic waves are enhanced into certain directions while are canceled in others, resulting in an amplified signal that is directed to certain positions. As they incorporate these main characteristics, directional antennas constitute an attractive component for all wireless devices.

Directional antennas can have many benefits in ad-hoc networks. The directional nature of the transmission results in spatial reuse as there can be multiple transmissions in the same neighborhood without the destruction of the transmitted packets. On the other hand, the directional transmission increases the signal energy towards the direction of the receiver resulting in the increase of the coverage area. These two benefits widely lead in the increase of the channel capacity.

Unfortunately, directional transmissions cause some serious problems in an ad-hoc environment. These problems are the increase of the instances of hidden terminal problem, the problem of deafness and the problem of the determination of neighbors' location. The first two problems are studied extensively in [2], while the third is a natural problem that arises from the fact that for the transmission of a packet, the transmitter must know the location of the receiver to turn over the beam to the appropriate direction.

Traditional MAC protocols [5],[6] that have been designed for adhoc networks with omnidirectional antennas are not suitable for the support of the new feature. For that reason there is a strong 
demand for the design of new protocols that will be efficient enough to take advantage of the benefits that are provided, while minimize the effects of the weaknesses.

The rest of this paper is organized as follows: in the next section we discuss previous related work. In section 3 we give a summary of 802.11 and our antenna model. We propose our MAC protocol that adapts directional antennas in ad-hoc networks in Section 4. Finally, in section 5 we compare the performance of our protocol with other MAC protocols trough simulation studies.

\section{RELATED WORK}

Although work on the designing of MAC protocols for ad-hoc networks using directional antennas is limited, several researchers have tried to address this issue. Zander in [15], has proposed to use directional antennas in slotted Aloha multihop packet radio networks. More recently, Nasipuri, Ye, You, and Hiromoto in [10], proposed a variation of RTS/CTS mechanism of IEEE 802.11 adapted for use with directional antennas. Their protocol sends the RTS and CTS packets omnidirectionally in order to enable the transmitter and receiver to locate each other and then sending the data packet and Ack in direct mode.

Ko, Shankarkuman and Vaidya [7] propose another MAC protocol that sends a directional RTS that is followed by an omni CTS. They assume that the transmitter knows the receiver's location, so it transmits directionally the RTS to it. They propose an alternative of that scheme in case of lack of information for the location of the receiver. In this case the RTS is transmitted in omni mode in order to seek the receiver. Takai, Martin, Ren and Bagrodia [14] propose Directional Virtual Carrier Sensing in which they use directional RTS and CTS transmission. For the operation of this scheme they assume that the receiver's location is known by the transmitter. In the opposite situation, they propose the omnidirectional transmission of RTS. They also propose a cache scheme where they maintain information about the location of their neighbors, which is updated every time a node receives a frame. Ramanathan in [12] studies some very interesting issues about the performance of ad-hoc networks with directional antennas and the factors that affect it. He simulates several schemes, giving very useful results about the effectiveness for each one of them. Finally, Choudhury, Yang, Ramanathan and Vaidya [2] present a study about the problems that appear using directional antennas and in parallel they propose a MAC protocol for multihop transmissions that uses a directional multihop RTS.

The studies we have just mentioned have some common characteristics that lead to inefficiencies of the proposed protocols. Papers [10],[7],[12] employ at least one omnidirectional transmission of a control packet, limiting in this way the coverage area. If there is even one omnidirectional frame in the handshake between transmitter and receiver, then the directional transmissions must be limited in the coverage of the omni range as the maximum distance between the two stations is defined by the smaller coverage range of the four frames of the handshake. Assume that the RTS transmission is in omni mode while the other three transmissions (CTS, DATA, ACK) are in directional mode. Then the directional mode transmissions must reduce their transmission energy, to cover no more than the coverage area of the RTS. This is clearly depicted in Figure 1 and constitutes a disadvantage of the mentioned MAC protocols as in this way they do not exploit one of the main benefits of the directional transmission, the increase of the coverage range.

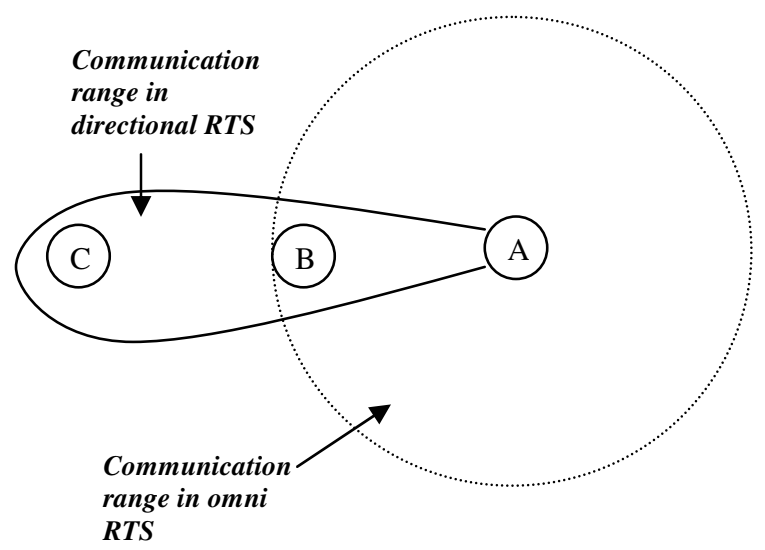

Figure 1: The coverage range of a MAC protocol that uses an omni RTS transmission and directional CTS, Data, Ack transmissions

As described in [3],[8],[4], with $M$ antenna elements and with the same transmission energy, an antenna array provides an increased antenna gain, in comparison with the omni mode of the order of $M$. This gain is doubled if there is directivity in both transmission and reception. So a directional communication between two stations can have a significant increase in the distance between them, compared with the equivalent omni communication, a benefit that it is not exploited at all by the above schemes.

In [2],[14] the use of only directional transmissions in the four way handshake, does not overcome main problems: the increase of instances of hidden terminal problem, the problem of deafness and the problem of determination of neighbors' location. The reasons why the first two problems arise are studied explicitly in [2]. The third problem arises from the fact that sending a directed RTS assumes that the information about the receiver's location is known or is given by another mechanism or from the above layer. This assumption simplifies the scheme a lot, making it unsuitable for implementation, without the contribution of an external mechanism, the design of which is not itself a trivial problem.

To be more accurate, the important information about the location of a node is not the location in its own right, but the direction in which the node must turn its beam in order to have access to its neighbor. So the knowledge of the position of a node (which [7] assumes by means of additional hardware as GPS) may not always be the right guide for the decision of the beam direction. There can exist a physical obstacle between receiver and transmitter, so the connectivity may happen by reflection of the radiowave, resulting in reception of the signal from a direction different of the neighbor's direction.

Finally [2],[7],[14] discuss the concept of the D-NAV, a directional Network Allocation Vector that adapts the concept of NAV that has been introduced by IEEE 802.11 into the directional environment. These studies contain an interesting, qualitative description of the D-NAV, without the functional details that must be defined to make its use beneficial. 


\section{PRELIMINARIES}

\subsection{IEEE 802.11}

IEEE 802.11 [5],[11] is a popular protocol that defines the functionality of MAC and PHY layers of a wireless add-hoc or infrastructure network. Here we summarize some aspects of it that are particularly important in the operation with directional antennas. In the case of MAC in ad-hoc, 802.11 introduces a Distributed Coordination Function (DCF) that coordinates the medium occupancy using CSMA/CA. A station must hear the medium and if it finds it idle for a predefined time (DFTS) it can start data transmission to the receiver. When the receiver hears the data packet responds with an acknowledgment (Ack) packet.

To deal with the problem of hidden terminal [1],[9] in wireless communications, an extra scheme, that use a handshake of a Request To Send (RTS) and Clear To Send (CTS) frames can be added to the basic transmission scheme (Data+Ack). This scheme is used to guarantee that the receiver is in a state capable of receiving a data packet. The transmitter informs the receiver for the intended transmission and if it receives a CTS immediately after its RTS it starts the transmission of data, otherwise is doing a back-off. In this way a four way handshake is created (RTS-CTSData-Ack) that is used for the avoidance of collisions.

All four frames contain information about the duration of the pending handshake, informing the neighbors to avoid starting a new transmission during this period. This is managed by a mechanism called Virtual Carrier Sense. In this mechanism, every station maintains a Network Allocation Vector (NAV). If NAV is equal to zero the station can transmit otherwise it can not. NAV is initially equal to zero. If a NAV is a positive number, there is a countdown until it reaches zero. When a station hears one of the four frames, it updates its NAV with the duration of the pending handshake, preventing itself by transmitting until its NAV reaches zero again. With this scheme every station performs a Virtual Carrier Sense in addition to the physical carrier sense to enhance the resistance of the protocol against collisions.

\subsection{Directional Antennas}

A directional antenna can transmit a signal in any direction, using an array of antennas called array of elements. Individual omnidirectional transmissions from these elements interfere constructively or destructively with each other, resulting in increase of signal strength in one or more directions and elimination in the others. The more the elements of a directional antenna are, the more the increase of the signal in the desired direction. There are directional antennas with 1 (omnidirectional) $2,4,8,16$ etc. elements. The interested reader can find an intensive study of directional antennas in [8],[13].

As the number of antenna elements increases, the beam width and the signal gain can be controlled more effectively. An important element of our protocol is covering the whole area around the transmitter with successive sequential transmissions. In the rest of the paper we will assume that we can provide effective omni transmission with $M$ sequential directional transmissions when we have $M$ antenna elements. In certain cases this number may need to be higher but our conclusions based on numerical experiments we performed will not change.

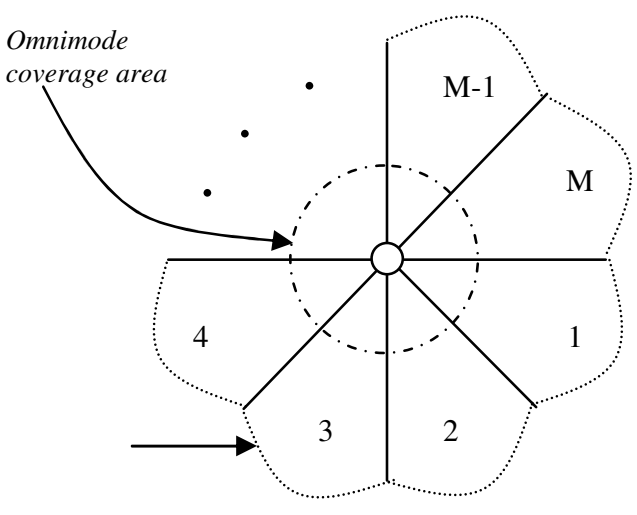

Figure 2: A node with $M$ beams

As we can see in Figure 2, the area around the node is covered by $M$ beams. We assume that the beams are not overlapping. We number the beams from 1 to $\mathrm{M}$ starting from the beam that is located just right of the 3' o clock position. The node can transmit its signal to anyone of the $M$ beams, increasing the coverage range of the transmission towards a specific direction. In idle mode the node hears omnidirectional. In the reception of a signal the node uses selection diversity, which means that it uses the signal from the antenna that is receiving the maximum power of the desired signal. With this mechanism the receiver can extend the communication area, which means that the communication link can benefit more by beamforming at both transmitter and receiver.

\section{THE PROPOSED PROTOCOL}

In the previous section we summarized the advantages, disadvantages and functional characteristics that appear in ad-hoc networks when directional transmission is used. These points can constitute a guideline for the intensification of the principles that must govern the design of a MAC protocol that will coordinate the communication in these environments. We are going to outline these principles in the following lines:

- An effective protocol must use only directional transmissions, to fully exploit the increase in coverage range with the use of directional antennas.

- There must be a mechanism that informs the neighbors to defer their transmission if this is going to harm the pending transmission, decreasing in this way the problem of hidden terminal.

- There must be a mechanism to deal with the problem of deafness.

- The proposed scheme must provide an efficient way identify the neighbors' location and to maintain this information for use in directional transmissions. This scheme must be simple and must exchange very short information between stations if this is necessary.

Proposed MAC protocols that use at least one omnidirectional control frame transmission [7],[10],[14] do not comply to the first principle, while they comply, in a sense, to the second and third principle. This happens due to the fact that the omni transmission of the control frame spreads around the information about intended transmissions. Regarding the fourth principle, although 
[7],[14] propose schemes for the solution of the neighbors' location problem, they do not give a detailed description. On the other hand, a part of [14] and the proposal MAC protocol in [2] observe the first principle, as they propose the use of directional transmissions for RTS-CTS but they do not deal with the problems of hidden terminal and deafness as it is described in detail in [2]. Furthermore, [2] assume the providence of neighbors' location information by an upper layer resulting in the lack of observation of the forth principle.

In the following paragraphs we are going to describe our proposed protocol. Our protocol has been designed under the guidance of the principles that we have just mentioned. It is simple in the implementation, as it is based in the concept of the IEEE 802.11 protocol. Nevertheless, it uses only directional transmissions, increasing in that way the coverage area. Moreover, the transmitter informs the neighbors about the intended transmission to defer their transmission if this is going to harm the initial one. Finally, our proposal neither assumes a priori any information about the neighbors' location nor depends on any information that receives for that reason from an upper layer as in [2]. It provides a simple scheme for recording and maintenance of neighbors' location exchanging between neighbors minimal information. Furthermore, this scheme assists the functionality of the DNAV scheme proposed in [7], making it simpler in implementation.

\subsection{Circular Directional RTS}

Our protocol is based in a simple and innovative scheme of RTS transmission. In this scheme the RTS is transmitted directional consecutively, in a circular way, until it scans all the area around the transmitter. The circular transmission of the CTS as well, is an alternative that may enhance the performance of the protocol. But because this elaboration may result in unpredictable behavior of the communicating stations, this is something that we will not study in this work. We are going to investigate this idea in our future studies.

As we have mentioned we assume antennas with predefined number of beams, $\mathrm{M}$ in the figure 2, that cover the area around the transmitter. The transmitter starts transmitting its RTS in a predefined direction, assume with beam 1. Short afterwards it turns its transmission beam on the right sending the same RTS with the next one (beam 2). It continues this procedure again and again until the transmission of RTS covers all the area around the transmitter (until it sends the RTS with beam M).

The RTS contains the duration of the intended four way handshake (as in 802.11). As this information is spread around by the circular RTS, the neighbors are informed about the intended transmission. The neighbors, after executing a simple algorithm that is described later in this section, decide if they will defer their transmission in the direction of transmitter or receiver, if this harms the ongoing transmission. In this way the neighbors are aware of the intended handshake, a fact that results in reduction of the hidden terminal problem.

The STA that is the destination of the RTS waits until the finish of the circular RTS transmission and afterwards it sends a directional CTS towards the direction of the transmitter of the RTS. On the other hand, when the transmitter completes the circular transmission of RTS, it hears the medium omnidirectional to receive this CTS. That means that the carrier sensing from the transmitter of the RTS in this phase is performed in an omnidirectional mode, just as in 802.11. If the CTS is received during a predefined period (CTS time out) then the transmitter continues with the transmission of the data packet and the reception of the Ack, just as in 802.11, except of the fact that now the transmissions of the Data and Ack packets are directional. By using only directional transmissions of RTS, CTS, Data, Ack, we exploit the benefit of increasing coverage area, compared with the case of at least one omnidirectional transmission that limits the coverage area in this of omni mode transmission.

It is important to mention that the transmitter and the receiver do not need any information about each other's location. The circular RTS reaches the target node wherever is located. On the other hand the receiver, using selection diversity, receives the signal from the right beam and so sends it's RTS by the same beam. Nevertheless, we propose a simple scheme for track and maintenance of other nodes' location that helps for a more efficient functionality of the network.

\subsection{Neighbors' location}

The difficult issue in the directional transmission of the four frames, in view of the lack of location's information, is the transmission of the RTS. If the receiver hears the RTS, using selection diversity, it will recognize the direction by which it receives the signal and so it will transmit the CTS in the right direction. Accordingly, the transmitter recognizes the direction of the receiver by the reception of the CTS and sends the Data packet in the right way. Our protocol guarantees the transmission of the RTS in the direction of receiver by the circular directional RTS. So, if the handshake is completed, transmitter and receiver will know each other's location. More precisely, each of them knows the beam by which it can reach the other. Our protocol exploits this information by a simple scheme to solve the nodes location problem.

According to that scheme, every node maintains a table, called Location Table, with one record for every station that it has heard. Initially the Location Table is empty and it is updated in every reception. Because of the mobility of the stations a record may be updated many times.

In every record the node maintains the following information: $\boldsymbol{M e}$ (itself), Neighbor (the station from which it has heard a packet), My beam (the beam from which the transmitter heard the packet), Neighbor's beam (the beam by which receiver sent the packet). In this way every station maintains pairs of beams that are used for a direct transmission.

Table 1: A record of Location Table maintaining in node A of Figure 3

\begin{tabular}{|c|c|c|c|}
\hline Me & Neighbor & My Beam & $\begin{array}{c}\text { Neighbor's } \\
\text { Beam }\end{array}$ \\
\hline A & B & 4 & 2 \\
\hline
\end{tabular}

The record of table 1, means that $\mathrm{A}$ can transmit or receive from $\mathrm{B}$ by beam 4, while B can transmit or receive from A by beam 2 . This is illustrated in Figure 3, where every station uses four beams. The information "A, 4" is known from A by the selection diversity mechanism. The problem is that A can not realize the 
beam by which $\mathrm{B}$ receives its packet. For that reason, in every packet, in addition to the other information, the transmitter sends a number which indicates the beam by which the packet is transmitted. In this way, by the reception of a frame the receiver can update all the information of the corresponding record.

As we have mentioned this information is not necessary for a four way handshake communication to take place, due to the circular directional RTS. Nevertheless, this information is useful in the decision of the neighbors about the deferment of their transmission or not, as we explain in the next session.

\subsection{Use of D-NAV}

As we have discussed, one of the main problems of the directional transmissions in ad-hoc networks is the increase of the hidden terminal problem. Our protocol deals with this problem informing the neighbors for the intended transmission. The neighbors receiving the circular RTS (or alternately the CTS) have to decide if it is necessary to defer their transmission in any specific location. In [14],[2] there is an extensive discussion about this problem. Both of these papers adopt the $\boldsymbol{D}-\boldsymbol{N A} \boldsymbol{V}$ scheme proposed in [7] as a mechanism to deal with this issue. D-NAV is a Directional Network Allocation Vector that functions in the philosophy of NAV proposed by IEEE 802.11, adapted in the directional communication.

D-NAV uses a table that keeps track of the directions and the corresponding durations, towards which the station must not initiate a transmission [2]. The continuous update of this table with the right information, in order to keep neighbors silenced towards the right direction during a transmission, is important both for dealing with the hidden terminal problem as well as for the spatial reuse.

The right update of D-NAV table has two issues to deal with. The first is the informing of the neighbors about the intended transmission. The second is the right knowledge of neighbors of transmitter's and receiver's location for the proper decision about the directions in which a neighbor must defer its transmission in order not to destroy the intended handshake.

Papers [14],[2] discuss qualitatively that issue, but they do not propose a practical algorithm for the right update of the D-NAV table. The authors of [2], by assuming the knowledge of a node for other nodes' location, simplify very much the problem making it trivial. Moreover, the directional transmissions decrease the spread of the information that is given by RTS - CTS exchange about the intended transmission.

In [14], the authors propose the setting of D-NAV towards the location of the station by which it receives RTS, CTS or Data packet. In that way a neighbor can not realize by the reception of RTS, the location of the intended receiver of the RTS. So it can only defer towards the location of the transmitter. Consequently, for the right update of the D-NAV in an intended transmission, a neighbor must hear both RTS and CTS packets. Otherwise it will update its D-NAV table only towards one of the two nodes and so it may destroy the transmission. (Please bear in mind that for a successful transmission there must be no collision in the receiver as well as in the transmitter of the Data packet because of the exchange of the handshake).

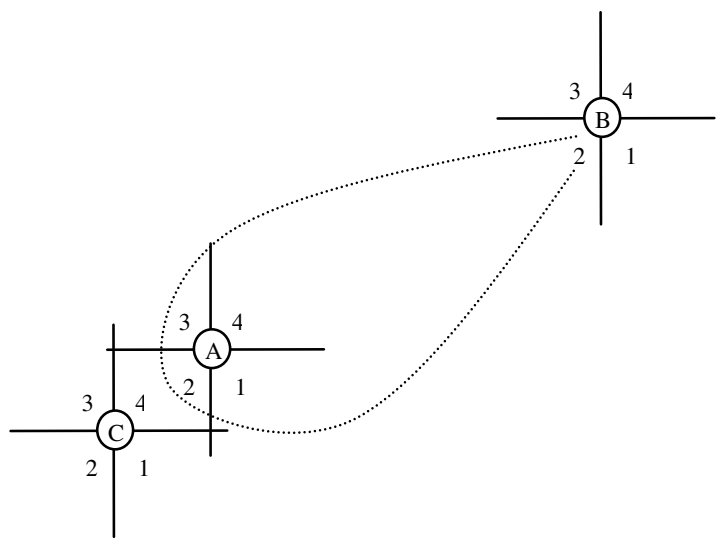

Figure 3: An example scenario of hidden terminal problem

One other problem that is discussed in [2] is the Hidden terminal problem due to asymmetry in gain. This problem is presented in Figure 3. Here node A sends RTS to B while C is a neighbor. When $\mathrm{A}$ and $\mathrm{C}$ are in idle mode they hear omnidirectional. As we can see B's beam can just reach A, making $\mathrm{C}$ a hidden node for B. In this scenario C can not hear A's RTS neither B's CTS. So C is not aware for the transmission of data frame from A to B. When A stars transmitting the Date frame directly to $\mathrm{B}, \mathrm{B}$ using selection diversity, receives directional too. If $\mathrm{C}$ sends a frame toward $\mathrm{B}$ 's location, while this transmission is in progress, B can hear this transmission due to its directional reception, confusing the two signals. This confusion results in packet collision.

Our protocol solves these problems by a very simple mechanism. As we have mentioned, every station maintains a Location Table. In this table, pairs of antenna beams are kept that interact for the communication between itself and its neighbors. When a station transmits an RTS or CTS to another station, the frame header, contains the corresponding beam pair. Every neighbor that receives one of these frames examines its Location Table to find the beams through which it can "see" the two stations. If one of these beams coincides with the respective beam of the RTS, CTS frame, then the neighbor defers its transmission by this beam. Let's see this mechanism by the example illustrated in Figure 4.

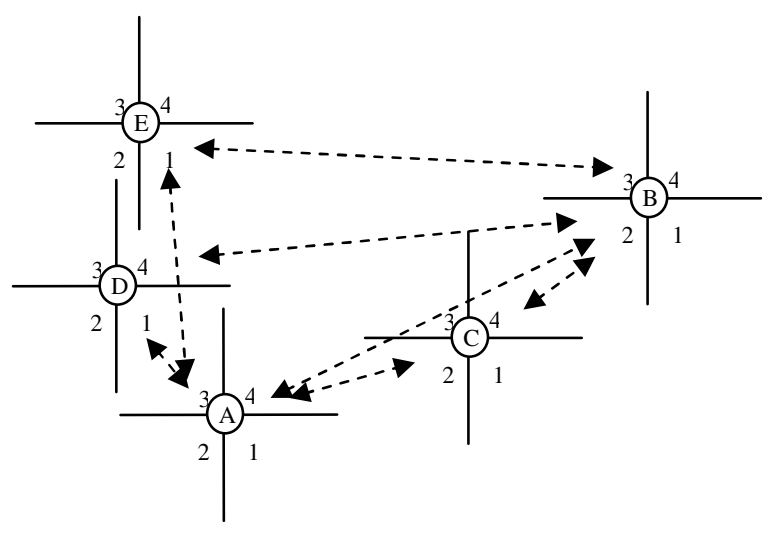

Figure 4: An examble scenario where nodes use their Location Tables 
In this figure, station A starts a transmission for station B. Station $\mathrm{C}, \mathrm{D}$ and $\mathrm{E}$ are neighbors that hear the circular RTS from A. The RTS as we can conclude by the figure will contain the following information: A, B, 4, 2. That means that A is going to send (RTS, Data) and receive (CTS, Ack) frames by beam 4, while B is going to send (CTS, Ack) and receive (RTS, Data) frames by beam 2 . From the figure we can see that the corresponding modules of the Location Tables in the three nodes are:

Table 2: Modules of Location Tables maintained by nodes C, D, E of Figure 4

For node C

\begin{tabular}{|c|c|c|c|}
\hline Me & Neighbor & My Beam & $\begin{array}{c}\text { Neighbor's } \\
\text { Beam }\end{array}$ \\
\hline C & A & 2 & 4 \\
\hline C & B & 4 & 2 \\
\hline
\end{tabular}

For node D

\begin{tabular}{|c|c|c|c|}
\hline Me & Neighbor & My Beam & $\begin{array}{c}\text { Neighbor's } \\
\text { Beam }\end{array}$ \\
\hline D & A & 1 & 3 \\
\hline D & B & 4 & 2 \\
\hline
\end{tabular}

For node $\mathrm{E}$

\begin{tabular}{|c|c|c|c|}
\hline Me & Neighbor & My Beam & $\begin{array}{c}\text { Neighbor's } \\
\text { Beam }\end{array}$ \\
\hline E & A & 3 & 3 \\
\hline E & B & 3 & 3 \\
\hline
\end{tabular}

By the information that is contained in the RTS, the three neighbors know that A will receive by beam 4 while $B$ will receive by beam 2 . Hence, every neighbor examines its Location Table (illustrated in Table 2) to see if it can destroy the reception in A or B. if this can happen, it defers its transmission by the corresponding beam updating its D-NAV table.

Lets see how C, D, E will act in the previous scenario. C recognizes that A can hear it by beam 4, while B can hear it by beam 2 . Thereby, it realizes that it can interfere in the reception of both $\mathrm{A}$ and $\mathrm{B}$. This information leads $\mathrm{C}$ to defer its transmission for the corresponding duration by the beams that can transmit this interference, namely beam 2 and 4, updating the D-NAV table.

D will recognize that A can hear it by beam 3, while B can hear it by beam 2 . Thereby, it realizes that it can interfere only with the reception of $B$ and so it defers its transmission through beam 4 updating its D-NAV table.

Accordingly, E realizes that it can not harm the intended transmission and so it does not defer its transmission by any beam.
It is worth to say that the tree nodes act in that way having heard only RTS from A. In previously proposed protocols due to the lack of the extra information that we have added in RTS, neighbors, receiving RTS, can not realize the beam by which B will receive the Data frame and so they will update their D-NAV table only with the information about the location of A, not protecting in that way the reception in B. They must hear CTS too, to realize B's location and hence to protect the reception in this node.

Let's see how our protocol will affect the collision of Figure 3. Node $\mathrm{C}$ will hear the circular directional RTS from A, being aware for the intended transmission. Reading the extra information into RTS about the Beams that will be used for this transmission, $\mathrm{C}$ will examine its location table and will defer the transmission toward B's location (i.e. beam 4) resulting in this way in the avoidance of the collision.

Our protocol supports in an efficient way the mobility of the stations, as the Location Table is updated very often (every time a node receives a packet) leading to a contentious tracing of neighbors position.

\subsection{Some protocol details}

There are some details related to the implementation of the protocol. These are the necessary changes in some time periods that have been defined in 802.11, in order to support our protocol. In the next paragraphs assume that $\mathrm{A}$ is the transmitter node and $\mathrm{B}$ is the receiver.

\section{CTS is transmitted after the circular RTS}

Now the CTS frame must be transmitted by B, just after the conclusion of the circular RTS. For this reason, B waits for time T (instead of SIFS in 802.11) after the reception of RTS and then it transmits the CTS. The time $\mathrm{T}$ is calculated as follows:

$T=k * R T S$ transmission time + SIFS, where $k=M-A$ 's beam number.

Thus, if the B receives an RTS by A by its first beam (Beam 1) and $\mathrm{M}=4$, it will wait for $(4-1) * R T S$ transmission + SIFS. This is the time needed for three more RTS transmissions until the conclusion of the circular RTS transmission. If $\mathrm{B}$ receives an RTS from A by its final beam (beam 4) then it will send CTS, SIFS time after RTS reception (as now $\mathrm{k}$ is 0 ).

During the waiting time T, B is locked in a "Ready for transmission" mode ignoring the reception of other packets.

\section{An idle node hears the channel for longer time than DIFS before transmission}

It is obvious that now every idle node must hear the channel more than DIFS before its transmission, as now RTS is circular and so its spread around a node takes more time compared with omni mode. For that reason in our simulations we defined an idle node to hear the medium for $M^{*} R T S$ transmission time before its transmission.

3. Duration field of RTS packet is decreased by RTS transmission period, every time an RTS frame is transmitted in the cycle. 
As we have mentioned, RTS informs neighbors about the intended data transmission. In 802.11 RTS duration field contains the time period that is needed for the conclusion of the whole handshake. That means

Duration period $=$ RTS trans time + SIFS + CTS trans. Time + SIFS + Data trans time + SIFS + Ack trans time.

Now the RTS transmission time depends on the beam that is to be sent. Thus for the first beam

Circular RTS trans time $=(M-1) *$ RTS trans. time

For the second beam

Circular RTS trans time $=(M-2) *$ RTS trans. time

Etc.

\section{The circular RTS respects the on going transmissions.}

If the D-NAV of A does not allow the transmission of the RTS towards a specific direction, A does not send the RTS towards this direction, remaining in silent mode during the corresponding period. In this way the transmitter respects the on going transmission.

\section{SIMULATIONS}

\subsection{Simulation Model}

To evaluate the performance of our MAC protocol, we developed an event driven simulator and performed simulations for various scenarios. In our simulations we assumed that the physical channel is error free and the propagation delay is zero. The destination of each packet is chosen randomly from the set of the station's neighbors. The packet length is constant and equal to 1024 bytes. The packet arrival at each station is a Poisson process with the same mean $\lambda$. To vary the load of the network we vary $\lambda$. In such a way we vary the offered load in the queue of every station. The value of $\lambda$ depends on the number of stations that participate in the scenario and the overall load we want to achieve. The bigger the number of stations is, the lower the offered load we set for every station, to achieve a specific value of the overall load. To decrease the offered load of every station we increase $\lambda$. On the other hand, to achieve a higher value of the overall load when the number of stations remains constant, we increase the offered load in every one of them, decreasing the value of $\lambda$.

The performance metric used to evaluate the protocols is the aggregate throughput achieved by the network. As throughput we define the percentage of the channel rate that is used for transmission of data packets. An overall throughput that exceeds $100 \%$ means that there is more than one pair of nodes that communicate simultaneously for a period of time. So such a result is an indication of the average channel reuse ratio due to the use of directional transmissions.

Each simulation runs for 200 seconds with a warm up period of 50 seconds. In our simulations we have used nodes that were equipped with antenna arrays of 1,4 and 8 elements. In the start of every simulation the Location Table of each station is empty and is gradually updated, as the simulation progresses.

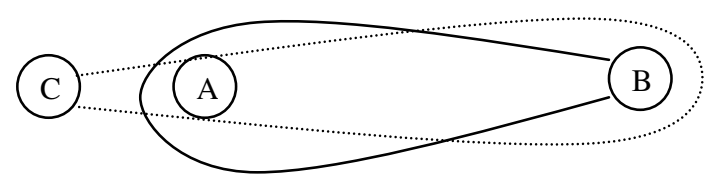

Figure 5: The transmissions of scenario in Figure 3.

\section{Simulation Results}

In our simulations we tried to study the benefits of our protocol, comparing it with other MAC protocols with omni and directional transmissions. More particularly, we compared it with 802.11 (that uses omni transmissions) as well as with D-MAC protocol that uses directional RTS and CTS transmissions, as this is described in [2]. We tried to ensure that the scenarios we have chosen guarantee equal conditions between the examined protocols. As a result, we simulate our protocol with D-MAC in specific scenarios with known topologies, as D-MAC assumes the knowledge of the location of the neighbors by any node. It makes no sense to compare these two protocols under lack of locations' knowledge conditions, as D-MAC would not operate properly. As the proposed protocol is the only integrated solution that uses directional transmissions and can operate without any assumptions about neighbors' location we compare it in arbitrary scenarios, where nodes have no knowledge for their neighbors' location, only with 802.11 .

We first evaluate the performance of the scenario demonstrated in Figure 3. We have selected this simple scenario to compare the effectiveness of our protocol with that of the D-MAC [7], which uses directional RTS-CTS. As we can see in Figure 5, A transmits to $\mathrm{B}$ while $\mathrm{C}$ transmits to $\mathrm{A}$. The coverage range of B's CTS does not include $\mathrm{C}$. On the other hand as $\mathrm{B}$ receives directionally towards A's direction it can hear simultaneous transmissions of A and $\mathrm{C}$ that lead to collision. The results are shown in Table 3.

As we have discussed in the previous session D-MAC comes short of throughput because of the hidden terminal problem due to the asymmetry in gain. Our protocol overcomes this problem, as $\mathrm{A}$ informs $\mathrm{C}$ for the intended transmissions by its circular RTS resulting in a throughput that is double the throughput of D-MAC.

Table 3: Simulation results of scenario in Figure 3 that study the hidden terminal problem in the proposed protocol due to asymmetry in gain.

\begin{tabular}{|c|c|c|}
\hline $\begin{array}{c}\text { Throughput (\%) } \\
\text { In high load }\end{array}$ & D-MAC & Our protocol \\
\hline Node A & 33.34 & 40.21 \\
\hline Node C & 15.57 & 39.89 \\
\hline Overall & $\mathbf{4 8 . 9 1}$ & $\mathbf{8 0 . 1}$ \\
\hline
\end{tabular}

In Table 3 we can also see that D-MAC has a fairness problem. Node $\mathrm{C}$ has almost half of the throughput of Node A. On the other hand, in our protocol, the overall throughput is shared equally between the two nodes (around 40\% for each one). This happens 
due to the fact that in D-MAC, node $\mathrm{C}$ can not be aware for the communication between A and B (as the RTS from A is directional towards B and the CTS from B can not reach C) so assuming that the medium is idle, it sends RTS again and again. As it does not receive a respond from A (A is communicating with B) it does back off again and again resulting in this way in large back off periods. Our protocol provides to $\mathrm{C}$ the information for the on going communication (by the circular RTS), resulting in the avoidance of these large back off periods.

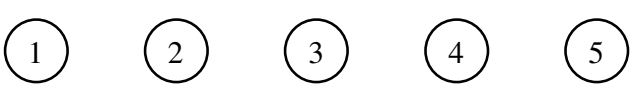

Figure 6: A scenario with linear topology

Our second experiment examines the overhead that is added by the circular transmission of the RTS. In the proposed protocol the transmission of the RTS lasts longer than in the directional or omni mode due to the fact that now RTS is transmitted M times.

By simulating the scenario of Figure 6 we measure the throughput degradation of our protocol in comparison with the directional transmission of RTS-CTS, in a case where our protocol does not benefit by its mechanism. In this scenario, as all nodes are in a linear topology, the transmission of the circular RTS is not necessary, as now all nodes can hear the directional RTS-CTS and so they can be informed for the intended transmissions. To see clearer the effect of the circular RTS we enhanced the directional RTS-CTS with the feature of informing the neighbors for the two stations that participates in the communication, just as in our protocol.

Table 4 shows the results of the simulation. As we can see, the proposed protocol has a degraded throughput of $3,5 \%$ in the case of $M=4$ and $7 \%$ for $M=8$. This overhead is due to the repeated transmissions of the RTS and is quite low in comparison with the benefits that arise by its use.

In our next experiment, we evaluate the throughput performance of a scenario with 7 nodes, randomly distributed in a two dimensional area. We examine the performance of our protocol compared with this of 802.11, as the offered load increases. We can see the topology of this scenario in Figure 7.

Table 4: Simulation results of scenario in Figure 6 that measure the effect of the circular RTS.

\begin{tabular}{|l|l|l|l|}
\hline & \multirow{2}{*}{$\begin{array}{l}\text { Enhanced } \\
\text { D-MAC }\end{array}$} & \multicolumn{2}{l|}{ Our Protocol } \\
\cline { 3 - 4 } & & $\mathbf{M = 4}$ & $\mathbf{M = 8}$ \\
\hline $\begin{array}{l}\text { Throughput (\%) } \\
\text { in high load }\end{array}$ & 169,11 & 163,54 & 157,4 \\
\hline $\begin{array}{l}\text { Degradation (\%) (compared } \\
\text { with Enhanced D-MAC) }\end{array}$ & $\mathbf{3 , 2 9}$ & $\mathbf{6 , 9 2}$ \\
\hline
\end{tabular}

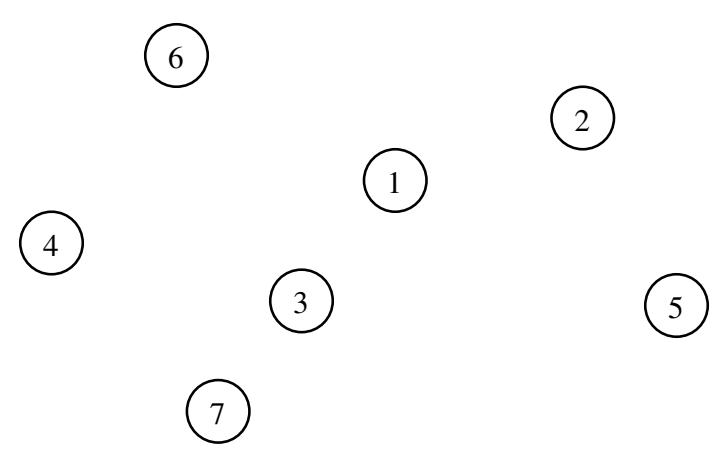

Figure 7: A random scenario with 7 nodes

In figure 7 , we assume that every node can hear the others. The coverage range of the three transmission models (antennas with 1 , 4,8 elements) is assumed to be equal. In this way we are going to measure the effect of the circular directional transmission of the RTS, keeping the others parameters constant. Please bear in mind that in the presented results we must add the extra enhancement of the throughput due to the increase in the coverage range.

The variation of the total throughput of the network with the total offered load is shown in Figure 8. As we can see the proposed protocol highly increases the throughput, as the number of antenna elements is increased. In heavy load conditions, the throughput of our protocol has a gain of $34 \%$ in the case of 4 elements and $42 \%$ in the case of 8 elements, compared with 802.11. In low load conditions, our protocol seems to have slightly lower throughput than 802.11. This happens due to the fact that in such conditions, directional transmissions can not benefit by the spatial reuse as the load is very low while the circular transmission of the RTS adds an extra overhead, compared with the omni transmission in 802.11. As the load increases, this extra overhead is quickly canceled by the increase of the throughput due to the spatial reuse.

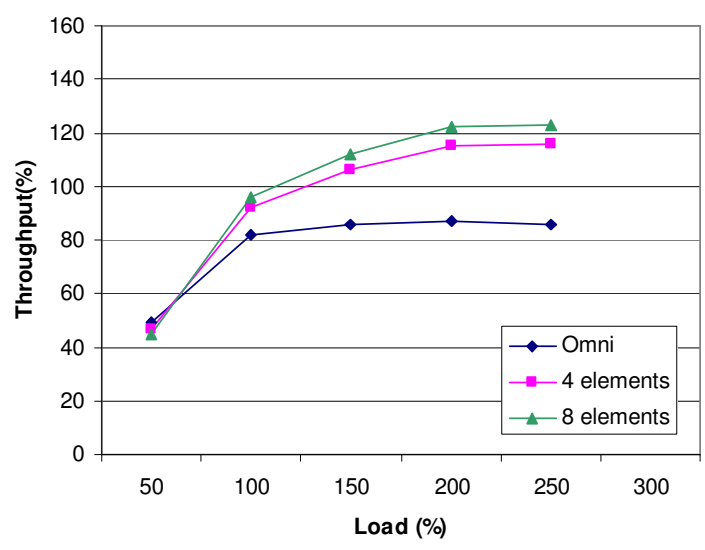

Figure 8: Throughput of the proposed protocol using antennas of 1, 4 and 8 elements for the arbitrary scenario of Figure 7. 


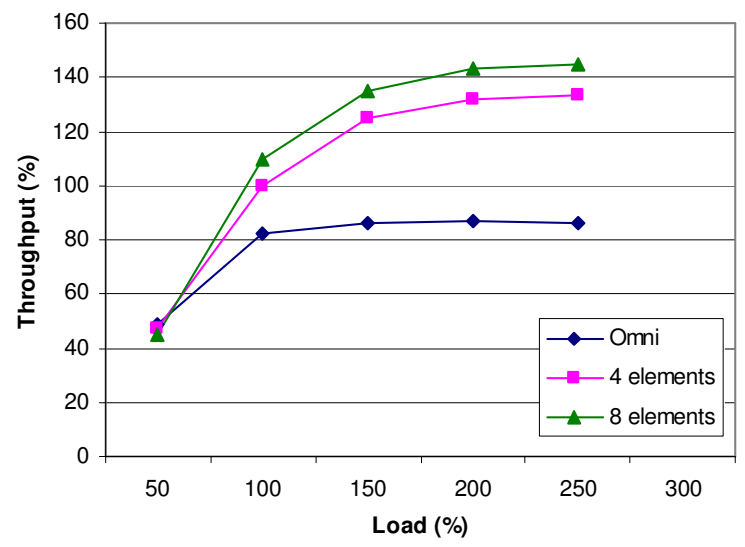

Figure 9: Throughput of the proposed protocol using antennas of 1, 4 and 8 elements for an arbitrary scenario of 15 stations.

Our next experiment is a generation of the previous scenario in a more arbitrary environment with more stations. We have used a topology with 15 stations random distributed in a two dimensional area. We have made the same assumptions again i.e. every node can hear the others and the coverage range of the three transmission models (antennas with 1, 4, 8 elements) is assumed to be equal. We have run the experiment for ten different arbitrary topologies of 15 stations. In Figure 9 we give the average results.

As we can see in Figure 9, the picture is proportional to this of the previous scenario. What is worth mentioning is that the overall throughput of 802.11 converges again to almost the same value. This is something we expect as there is no spatial reuse in both scenarios of 802.11 because every station can hear the others. On the other hand, the throughput of our protocol increases compared with this in the situation of 7 stations. This happens because as in this scenario there are more stations, there is a higher probability of simultaneous transmissions between pairs of stations a fact that increases the spatial reuse.

Our final experiment uses the scenario in Figure 10. There are 9 nodes in a grid topology. The neighbors of the nodes can be concluded by the circular range around station 5 that depicts the coverage range of the 8 beams of this station.

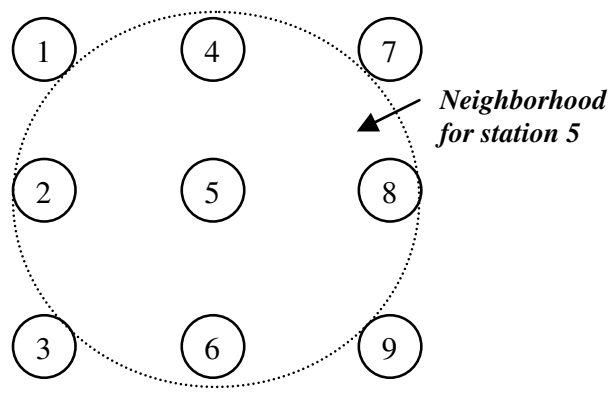

Figure 9: A grid topology scenario

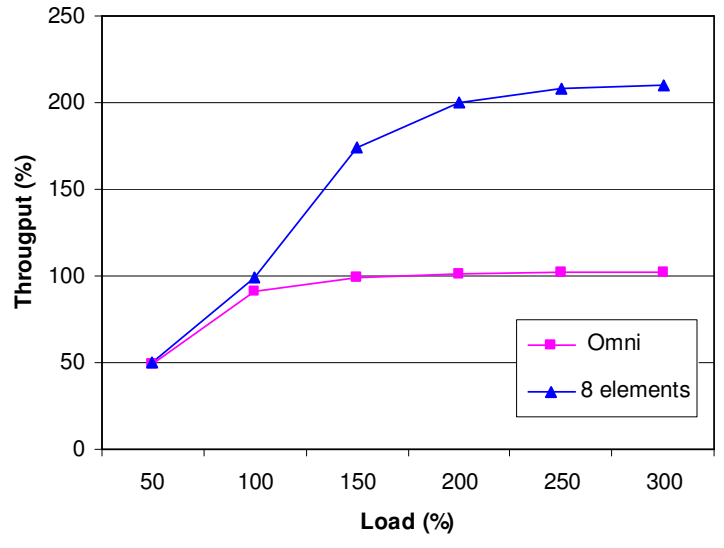

Figure 10: Throughput of the proposed protocol for the grid topology of Figure 9.

The results of the simulations are shown in Figure 11. Our protocol performs better than 802.11 as the offered load increases. In heavy load conditions the throughput achieved by the proposed scheme is double the throughput of 802.11. This happens due to the fact that spatial reuse is stronger as the load increases. On the other hand on the case of 802.11 , the hidden terminal problem and the exposed terminal problem (where there are differs in nodes that do not destroy any transmission) do not enable a high level spatial reuse.

\section{CONCLUSIONS}

In this work we propose a MAC protocol suitable of networks with directional antennas. Our protocol, utilizing a new scheme for the broadcasting of RTS, employs only directional transmissions, increasing in this way the coverage area. Particularly, the proposed algorithm is based in a circular, directional RTS that scans the area around the transmitter, informing the neighbors for the intended communication. Using a simple as much as effective scheme, the neighbors decide for their transmission differentiation in order not to destroy the on going transmission. In this way, there is a strong decrease in the hidden terminal problem. The protocol does not assume any knowledge of the neighbors' location. The previous features result in an efficient, integrated scheme that can be implemented easily.

\section{ACKNOWLEDGEMENTS}

Thanasis Korakis' research work is partially supported by the Greek State Scholarship's Foundation (IKY).

\section{REFERENCES}

[1] Chane L. Fullmer, J. J. Garcia-Luna-Aceves, "Solutions to Hidden Terminal Problems in Wireless Networks", SIGCOMM '97.

[2] R.R. Choudhury, X. Yang, R. Ramanathan, N. H. Vaidya "Using Directional Antennas for Medium Access Control in Ad Hoc Networks", ACM MobiCom 2002, September 2002. 
[3] M. Cooper and M. Goldburg "Intelligent Antennas: Spatial Division Multiple Access", Annual Review of Communications, pages 999-1002, 1996

[4] G.J. Foschini, M.J. Gans, "On limits of wireless communications in a fading environment when using multiple antennas", Wireless Personal Communication 1998.

[5] IEEE 802.11, "Part 11: Wireless LAN Medium Access Control (MAC) and Physical Layer (PHY) Specifications", IEEE 802.11 Std, Aug 1999

[6] P. Karn, "MACA - A New Channel Access Method for Packet Radio" In Proc. 9th ARRL Computer Networking Conference (1990)

[7] Y.B. Ko, V. Shankarkumar, and N.H. Vaidya, "Medium access control protocols using directional antennas in ad hoc networks", In Proceedings of IEEE Conference on Computer Communications (INFOCOM), volume 1(3), pages 13-21, Tel Aviv, Israel, Mar. 26-30 2000.

[8] C. Liberti and T.S. Rappaport, "Smart Antennas for Wireless Communications: IS-95 and Third Generation CDMA Applications" Prentice Hall, April 1999.
[9] W.M.Moh, D.Yao and K.Makki, "Wireless LAN: Study of hidden terminal effect and multimedia support", Proc. Computer Communications and Networks, pp.422-431, October 12-15, 1998.

[10] A. Nasipuri, S. Ye, J. You, R.E. Hiromoto, “A MAC protocol for mobile ad-hoc networks using directional antennas", In Proceedings of IEEE Wireless Communications and Networking Conference (WCNC), Chicago, IL, Sep. 23-28 2000.

[11] Bob O'Hara, Al Petrick, “The IEEE 802.11 Handbook, A Designer's Companion", IEEE Press, 1999

[12] R.Ramanathan, "On the performance of Ad Hoc Networks with Beamforming Antennas", ACM MobiHoc, October 2001.

[13] T. S. Rappaport, "Wireless Communications, Principles and Practice", Prentice Hall, 1996

[14] M. Takai, J. Martin,A. Ren, R. Bagrodia "Directional Virtual Carrier Sensing for Directional Antennas in Mobile Ad Hoc Networks", ACM MobiHoc, June 2002.

[15] J. Zander, "Slotted ALOHA multihop packet radio networks with directional antennas," Electronics Letters, vol. 26, no. 25, 1990. 